Host-cell apoptosis in Taenia solium-induced brain granulomas in naturally infected pigs

Sikasunge, C.S.; Phiri, I.K.; Johansen, Maria Vang; Willingham lii, Arve Lee; Leifsson, Pall Skuli

Published in:

Parasitology

DOI:

$10.1017 / \mathrm{s} 0031182008004678$

Publication date:

2008

Document version

Publisher's PDF, also known as Version of record

Citation for published version (APA):

Sikasunge, C. S., Phiri, I. K., Johansen, M. V., Willingham lii, A. L., \& Leifsson, P. S. (2008). Host-cell apoptosis in Taenia solium-induced brain granulomas in naturally infected pigs. Parasitology, 135(10), 1237-1242. https://doi.org/10.1017/s0031182008004678 


\title{
Host-cell apoptosis in Taenia solium-induced brain granulomas in naturally infected pigs
}

\author{
C. S. SIKASUNGE ${ }^{1}$, I. K. PHIRI ${ }^{1}$, M. V. JOHANSEN²*, A. L. WILLINGHAM III ${ }^{3}$ \\ and P. S. LEIFSSON ${ }^{4}$ \\ ${ }^{1}$ School of Veterinary Medicine, University of Zambia, P.O. Box 32379, Lusaka, Zambia \\ ${ }^{2}$ DBL - Centre for Health Research and Development, Department of Veterinary Pathobiology, Faculty of Life Sciences, \\ University of Copenhagen, Thorvaldsensvej 57, DK-1870 Frederiksberg C, Denmark \\ ${ }^{3}$ WHO/FAO Collaborating Centre for Parasitic Zoonoses, Faculty of Life Sciences, University of Copenhagen, \\ Dyrlagevej 100, 1870 Frederiksberg C, Denmark \\ ${ }^{4}$ Department of Veterinary Pathobiology, Faculty of Life Sciences, University of Copenhagen, Ridebanevej 3 , \\ 1870 Frederiksberg C, Denmark
}

(Received 8 February 2008; revised 22 April and 27 May 2008; accepted 27 May 2008; first published online 14 July 2008)

\section{SUMMARY}

To assess whether apoptosis occurs in pig brain granulomas due to Taenia solium cysticerci, brain tissues from 30 pigs naturally infected with $T$. solium cysticercosis were evaluated by terminal deoxynucleotidyl transferase-end labelling (TUNEL) staining. In addition, tissues were stained with CD3 marker to identify T lymphocytes. Examination of TUNEL-stained tissues showed apoptotic cells in early lesions that contained viable cysticerci. Apoptotic cells were primarily found interspersed with normal cell types, and were mostly located in the inflammatory infiltrate. Late or advanced granulomas with disintegrated scolices did not show TUNEL-positive cells. CD $3+$ cells were found in both early and advanced lesions and apoptosis mainly co-localized with CD3 + T lymphocytes. This suggests that these cells are constantly undergoing apoptosis and thus die as soon as they arrive at the site of infection. Apoptosis indeed may be one way by which $T$. solium cysticerci down-regulate the host's cellular immune response in early cysticercosis. Therefore, further research is needed to establish if other cells besides T-lymphocytes are also a target for destruction by cysticerci in early cysticercosis as well as studies to assess if cysteine protease is expressed by viable cysticerci in situ.

Key words: Taenia solium, cysticercus, apoptosis, TUNEL staining, CD3 lymphocytes, granuloma, pig.

\section{INTRODUCTION}

Taeniosis/cysticercosis due to Taenia solium is a major parasitic disease of global proportions that poses serious threats to human health and economy in the third world countries (Sciutto et al. 2000; Garcia et al. 2005). The parasite's life cycle involves the pig as an intermediate host, harbouring larval stages called cysticerci and humans as the only known definitive host harbouring the adult tapeworm in the intestines. Humans can also acquire larval infection after accidental ingestion of human faeces contaminated with $T$. solium eggs, which evolves into human cysticercosis or neurocysticercosis (NCC) when the larvae lodge in the central nervous system (Flisser, 1994).

The nature of the immune response around the viable or degenerating cysticerci is vital in

* Corresponding author: DBL-Centre for Health Research and Development, Department of Veterinary Pathobiology, Faculty of Life Sciences, University of Copenhagen, Thorvaldsensvej 57, DK-1870 Frederiksberg C, Denmark. Tel. +45353314 38. Fax: +4535331433. E-mail:mvj@life.ku.dk understanding the underlying pathology in NCC (Uddin et al. 2006). The term 'immune tolerance' is used in cases of NCC where viable cysts without inflammation or surrounded by scanty inflammatory cells are observed (Carpio et al. 1998). Living parasites without inflammation are found more frequently in asymptomatic and immunodeficient patients whereas severe inflammation has only been seen in symptomatic patients (White et al. 1995). The limited inflammatory reaction in NCC could be induced by parasite apoptosis that leads to clonal deletion of both thymocytes and mature $\mathrm{T}$ cells (Murphy et al. 1990; Lenardo, 1991). Apoptosis is a type of programmed cell death, and an active process associated with distinctive biochemical and morphological changes that can be elicited by different triggers, including serum deprivation, and by extracellular stimuli acting through cell-surface receptors (Behnia et al. 2000; Silva et al. 2005).

Taenia solium cysticerci have been reported to employ various strategies to maintain a Th2 permissive environment (Villa and Kuhn, 1996). Cysticerci of $T$. solium are known to secrete in vitro a factor that depresses human and murine lymphocyte proliferation (Tato et al. 1995 ; Molinari et al. 2000) 
and that this factor impairs the humoral and cellular responses to metacestode antigens and inhibits the inflammatory reaction around metacestodes implanted subcutaneously in mice (Molinari et al. 1998). The ability of the cysticerci to kill host lymphocytes could enhance parasite survival. It is therefore possible that the parasite may be directly affecting host lymphocytes. This may be through reducing the functional activity of the cells, which is advantageous to the parasite, and apoptosis rather than necrosis would reduce inflammation (O'Connell and Rogan, 2000). In this study, we report for the first time, apoptosis in the inflammatory reaction in porcine brain tissues fixed in formalin and paraffin embedded.

\section{MATERIALS AND METHODS}

\section{Infected animals}

A total of 35 pigs were used in this study. Thirty pigs naturally infected with $T$. solium were purchased from the Livestock market in Lusaka and 5 healthy T. solium-free pigs from a commercial piggery in Lusaka. Pigs that were found to be positive for $T$. solium cysticercosis on tongue examination met the selection criteria into the study.

\section{Histopathological tissues and TUNEL staining}

Pigs were euthanized using Euthasol ${ }^{\circledR} \quad 20 \%$, intravenously $(200 \mathrm{mg} / \mathrm{ml}) \quad$ (Produlab Pharma, Raamsdonksveer, Netherlands) to effect and immediately bled. Specimens containing cysticerci and their surrounding tissue were collected from the brain of all 35 pigs. The cysts and surrounding tissue were fixed in $10 \%$ formalin for $12-24 \mathrm{~h}$ and then transferred to $70 \%$ ethanol and thereafter paraffin embedded. Five $\mu \mathrm{m}$ thick sections (Londoño et al. 2002) were mounted on positively charged slides (Fisher Scientific, Pittsburg, PA).

The terminal deoxynucleotidyl transferase-end labelling (TUNEL) staining was performed using the TdT-FragEL ${ }^{\text {TM }}$ DNA Fragmentation Detection Kit (QIA33, Calbiochem/Oncogene, Denmark) according to the manufacturer's instructions. Briefly, slides were deparaffinized to TBS. The area immediately surrounding the specimen was dried. All washing steps were done 3 times in Tris-buffered saline (TBS) and incubations were done at room temperature unless specified. Then $100 \mu \mathrm{l}$ of proteinase $\mathrm{K}$ diluted $1: 200$ in TBS were added to each specimen and incubated for $20 \mathrm{~min}$, after which the slides were washed. To inactivate the endogenous peroxidases, $100 \mu \mathrm{l}$ of $30 \% \mathrm{H}_{2} \mathrm{O}_{2}$ were added on each specimen and incubated for $5 \mathrm{~min}$. The slides were washed, following which they were incubated with $60 \mu \mathrm{l}$ of equilibration buffer for $20 \mathrm{~min}$. Fragment End Labeling (FragEL ${ }^{\mathrm{TM}}$ ) of DNA was done by addition of $60 \mu \mathrm{l}$ of TdT-enzyme and slides incubated for $1 \mathrm{~h} 30 \mathrm{~min}$ at $37^{\circ} \mathrm{C}$. The slides were washed and then incubated with stop buffer for $5 \mathrm{~min}$ before being washed again. Thereafter, the slides were incubated with a blocking buffer $(50 \mu$ l per specimen) for $10 \mathrm{~min}$, following which they were incubated with $60 \mu \mathrm{l}$ of streptavidin-horseradish peroxidase (HRP) conjugate diluted at $1: 50$ in blocking buffer for $30 \mathrm{~min}$. After washing, slides were incubated with $100 \mu \mathrm{l}$ of diaminobenzidine (DAB) solution for $10 \mathrm{~min}$. The resulting brown colour, after slides were washed, was counterstained with $0 \cdot 3 \%$ methyl green for $3 \mathrm{~min}$. To remove the excess dye, slides were washed in absolute ethanol 3 times, after which they were dried at $37^{\circ} \mathrm{C}$ and mounted with cover-slips for microscopical examination.

\section{Staining for CD3 + lymphocytes}

Tissue slides were deparaffinized to distilled water. All washing steps were performed twice in TBS, with each wash lasting for $5 \mathrm{~min}$. Incubations were done at room temperature unless stated otherwise. Epitopes were retrieved by microwave oven heating (Taylor et al. 1996; Pileri et al. 1997) at $700 \mathrm{~W}$ for $10 \mathrm{~min}$ in a buffer containing $0.01 \mathrm{M}$ Tris-base, $0.0005 \mathrm{M}$ ethylene glycol-bis(a-aminoethyl ether)-N,N,N,Ntetraacetic acid pH 9 ((EGTA) Sigma-Aldrich \#E3889) and leaving the sections in the hot buffer for $15 \mathrm{~min}$. The slides were then washed. Blocking of endogenous peroxidases was done by incubating slides with $100 \mu \mathrm{l}$ of $0 \cdot 6 \% \mathrm{H}_{2} \mathrm{O}_{2}$ in TBS for $15 \mathrm{~min}$. After washing, the slides were blocked for $30 \mathrm{~min}$ with $100 \mu \mathrm{l}$ of $20 \%$ normal goat serum (DAKO $\mathrm{X} 0907)$ diluted in TBS. Incubation with primary antibody CD3e-UNLB (SB 4510-01) diluted $1: 1000$ in $\mathrm{TBS} / 0 \cdot 1 \% \mathrm{BSA}$ was done at $4{ }^{\circ} \mathrm{C}$ overnight. The slides were then washed, after which they were incubated with EnVision/HRP (DAKO $\mathrm{K} 4001$ ) for $30 \mathrm{~min}$. After washing, slides were incubated with $100 \mu \mathrm{l}$ of DAB solution for $6 \mathrm{~min}$, then washed and counterstained with Mayers hæmatoxylin for $10 \mathrm{sec}$. Slides were washed with tap water and then distilled water and finally mounted with glycerol-gelatine for microscopical examination.

\section{Classification of cyst viability in brain granulomas}

Cysticerci were classified as either viable or calcified (dead) according to their microscopical appearance. Viable cysticerci had well-limited thin cystic wall structures containing scolices characterized by little or no inflammatory infiltrate. Calcified cysticerci (late or advance granulomas) were those with caseous amorphous material with disintegrated cyst wall and without discernible parasite structures and heavy inflammatory infiltrate. 

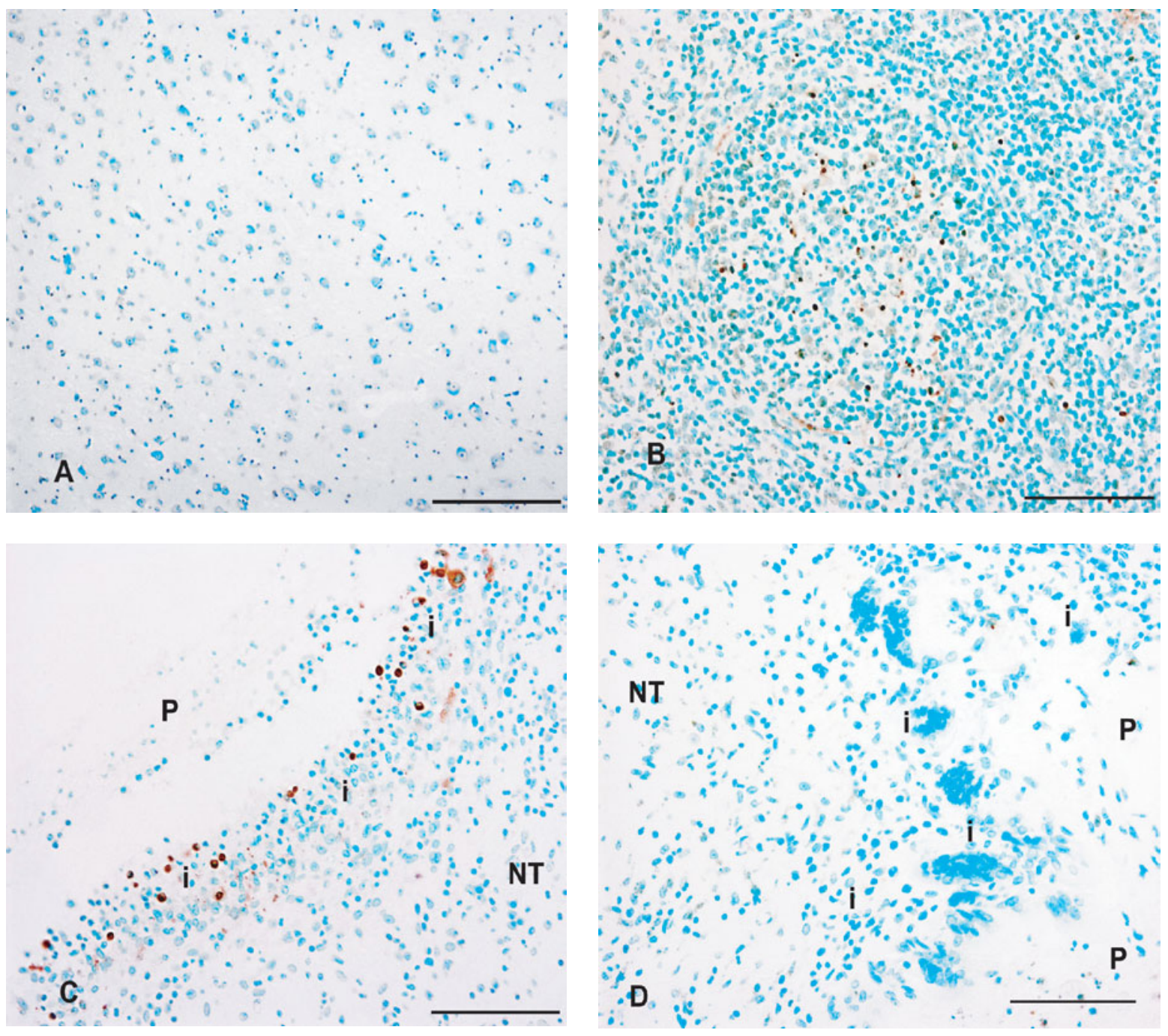

Fig. 1. TUNEL staining of porcine brain tissues naturally infected with Taenia solium cysticercosis. P, parasite; i, inflammatory infiltrate; NT, nervous tissue. (A) Normal nervous tissue, negative control. (B) Porcine lymph node showing tunnel-positive cells in germinal centre, positive control. (C) Early lesion. (D) Advanced lesion with dead cysticerci. Scale bar $=100 \mu \mathrm{m}$.

\section{Co-localization of CD3+ and TUNEL-positive cells}

In order to demonstrate co-localization of $\mathrm{CD} 3+$ cells and TUNEL-positive cells, 2 consecutive sections were cut from each paraffin block to be examined. Before the sections were mounted on slides, 1 from each pair was turned upside-down to ensure that the surfaces viewed at microscopy were 2 opposing surfaces. The sections were stained for CD3 + cells and TUNEL, respectively, as described above. The same areas with positive reaction were identified in each section from every pair using vessels and neurons as landmarks.

\section{RESULTS}

\section{TUNEL and CD3 staining}

Of the 30 pig brains with cerebral cysticercosis, 27 had viable cysticerci only while the remaining 3 had both viable and calcified cysticerci. Calcified cysticerci were only observed on the periphery of the cerebral cortex and none were observed in the medulla. Five specimens from each stage of the infection (viable $v s$ calcified) were further analysed by TUNEL staining. As expected, uninfected brain tissue was negative to the TUNEL staining (Fig. 1A). Porcine lymph node (Fig. 1B) was used as a positive control because in lymphoid organs, apoptosis occurs as a normal physiological process (Resendes et al. 2004). However, light microscope examination of TUNEL-stained tissues containing cysticerci confirmed that apoptotic cells corresponded to small, rounded dark-brown stained cells with the characteristic condensation and fragmentation of nuclear chromatin and loss of cytoplasm (Fig. 1C). Apoptotic cells were usually found interspersed with normal cell types, and were mostly located in the inflammatory infiltrate. TUNEL-positive cells were only concentrated in inflammatory infiltrates adjacent to viable cysticerci. Late or advanced granulomas with disintegrated scolices (Fig. 1D) did not show TUNEL-positive cells. 



Fig. 2. CD3 staining of porcine brain tissues from pigs naturally infected with Taenia solium cysticercosis. P, parasite; i, inflammatory infiltrate; NT, nervous tissue. (A) Normal nervous tissue, negative control. (B) Early granuloma. (C) Late granuloma. (D) CD3 + cells co-localization with TUNEL-positive cells. Scale bar $=100 \mu \mathrm{m}$.

In normal brain tissue, no $\mathrm{CD} 3+$ cells were detected (Fig. 2A). Brain granulomas induced by T. solium cysticerci showed CD3 + cells in both early and advanced lesions (Fig. 2B and C). Many CD3 + cells associated with viable parasite were present in clusters in the inflammatory infiltrate adjacent to the parasite. However, the abundance of $\mathrm{CD} 3+1 \mathrm{ym}-$ phocytes was markedly increased in advanced lesions and apoptotic cells co-localized mainly with CD3 + lymphocytes (black spots) (Fig. 2D). The co-localization showed that almost all apoptotic cells were CD3 positive but not all CD3 + cells were apoptotic.

\section{DISCUSSION}

To our knowledge this is the first report of apoptosis in porcine brain tissues infected with $T$. solium cysticerci that have been fixed in formalin and paraffin embedded. We have shown that $T$. solium cysticerci trigger apoptosis or programmed cell death in porcine brain granulomas. Of interest was the observation that the TUNEL staining detected apoptotic cells only in inflammatory infiltrate associated with viable parasite. Apoptosis of CD3+ lymphocytes observed in areas adjacent to T. solium cysticerci suggests that these cells are constantly undergoing apoptosis and thus die as soon as they arrive at the site of infection.

Apoptotic cells were usually found interspersed with normal cell types, and were mostly located in the inflammatory infiltrate, indicating that the events of apoptosis probably and only affect a certain percentage of cells (including CD3 + lymphocytes) in the inflammatory reaction in early granulomas when the cysticerci are alive. These observations are consistent with other patterns of apoptosis described by Presas et al. (2005), in which apoptotic cells were interspersed with normal cells in differentiating tissues of adult $T$. solium and $T$. crassiceps strobilae.

Our study showed evidence of apoptosis in early granulomas when the parasite was alive, while advanced and late granulomas were devoid of apoptotic cells. Hence, in the early stage, the cysticercus is able to evade the host's immune response, but this is lost 
in the late stage when the cysticercus dies. This can be explained by the fact that the evading ability in disintegrating parasites is absent, and this provides a favourable condition to the host, and allows the establishment of cell immune responses leading to granuloma formation and eventual parasite removal.

Lee and Piedrahita (2002) noted that apoptosis is an important aspect to be considered during hostparasite interactions given that bacteria, viruses and protozoa can regulate the death programmes of their hosts by activating, delaying or even preventing them. During host-parasite apoptosis, the rapid endocytosis of apoptotic bodies by phagocytic cells may prevent inflammation and excessive tissue damage (De Souza et al. 2008). Although it cannot be considered as the main type of cell death present in inflammatory reactions, parasite-induced apoptosis and its modulation by pro- and anti-apoptotic molecules (De Souza et al. 2008), may play a role in regulating the inflammatory response in early cysticercosis.

CD3 is a phenotypic marker that recognizes $\mathrm{T}$ cells. $\mathrm{T}$ cells are involved in cell-mediated immunity. They are essential in determining B cell antibody class switching, in the activation and growth of cytotoxic $\mathrm{T}$ cells, and in maximizing bactericidal activity of phagocytes such as macrophages (Koffeman et al. 2007). It is probably this diversity in function that makes the $\mathrm{T}$ cell subgroup of lymphocytes a target for destruction by $T$. solium cysticerci. Whether CD3 + lymphocytes were the only cell types undergoing apoptosis in the inflammatory reaction could not be evaluated in this study as we did not search for other cell types. Nevertheless, our results suggest that in cysticercosis, the cysticerci have suppressive effects on the host immune responses through destruction of T cells.

The mechanism by which $T$. solium cysticerci induce apoptosis in CD3 + lymphocytes, as found in this study, and CD4 + T-cells as reported by Solano et al. (2006) is still beyond the scope of many researchers of T. solium infections. Nevertheless, there are speculations that cysticerci may either excrete/ secrete or express factors which can induce apoptosis directly, or induce cells to excrete/secrete, which in turn causes cells to apoptose (O'Connell and Rogan, 2000). However, in Trypanosoma cruzi infections, the interactions between Fas ligand and Fas receptor (Lopes et al. 1999), as well as nitric oxide (Martins et al. 1999) has been linked to lymphocyte apoptosis induced by the parasite. Inhibition of caspase was reported to reduce lymphocyte apoptosis and improve host immune responses to infections caused by T. cruzi (Silva et al. 2007). Similarly, analysis of parasite-induced apoptotic $\mathrm{T}$ cells in skin-stage schistosomula of Schistosoma mansoni infection showed increases in the expression of Fas, FasL, and the Fas-associated death domain (Chen et al. 2002).
Our study carried out in porcine brain tissues has complemented the work conducted by Solano et al. (2006) who cultured lymphocytes with $T$. solium metacestode products. Our conclusions are that, $T$. solium cysticerci appear to induce death of CD3+ T-lymphocytes in the inflammatory reactions and that whatever was causing cell death did not affect all cells. Our results further suggested that any apoptotic effects would act locally since not all cells were killed, and the neuronal cells immediately surrounding cysticerci were not apoptotic. Therefore, further research is needed to establish whether other cells besides T-lymphocytes are also targets for destruction by cysticerci in early cysticercosis as well as studies to assess whether cysteine protease is expressed by viable cysticerci in situ.

We are grateful to the DBL-Centre for Health Research and Development for financial support. The support from the School of Veterinary Medicine, University of Zambia is also acknowledged. Special thanks go to Lisbet Kiørboe, Hanne H. Møller, Betina Andersen and Dennis Brok of the Section of Pathology in the Department of Veterinary Pathobiology, Faculty of Life Sciences, University of Copenhagen, Denmark, for their technical assistance with immunohistochemistry and photography. We would also like to thank Messrs P. Zulu and B. Chulu of the School of Veterinary Medicine, University of Zambia for taking care of experimental animals and J. Kaumba, M. Masuku, M. Chembensofu and M. Kankomba for their assistance rendered during the dissection work. Drs Jørgen Kurtzhals and Lothar Wiese are thanked for assistance with apoptosis staining.

\section{REFERENCES}

Behnia, M., Robertson, K. A. and Martin, W. J. (2000). Lung infections: role of apoptosis in host defense and pathogenesis of disease. Chest 117, 1771-1777.

Carpio, A., Escobar, A. and Hauser, W. A. (1998). Cysticercosis and epilepsy: a critical review. Epilepsy 39, 1025-1040.

Chen, L, Rao, K. V., He, Y. X. and Ramaswamy, K. (2002). Skin-stage schistosomula of Schistosoma mansoni produce an apoptosis-inducing factor that can cause apoptosis of T cells. Fournal of Biological Chemistry 277, 34329-34335.

De Souza, E. M., Meuser-Batista, M., Batista, D. G., Duarte, B. B., Araújo-Jorge, T. C. and Soeiro, M. N. (2008). Trypanosoma cruzi: Alpha-2-macroglobulin regulates host cell apoptosis induced by the parasite infection in vitro. Experimental Parasitology 118, 331-337.

Flisser, A. (1994). Taeniosis and cysticercosis due to Taenia solium. Progress in Clinical Parasitology 4, 77-115.

Garcia, H. H., Del Brutto, O. H., Nash, T. E., White, A. C., Tsang, V. C. and Gilman, R. H. (2005). New concepts in the diagnosis and management of neurocysticercosis (Taenia solium). American Fournal of Tropical Medicine and Hygiene 72, 3-9.

Koffeman, E., Keogh, E., Klein, M., Prakken, B. and Albani, S. (2007). Identification and manipulation of 
antigen specific $\mathrm{T}$-cells with artificial antigen presenting cells. Methods in Molecular Medicine 136, 69-86.

Lee, C. K. and Piedrahita, J. A. (2002). Inhibition of apoptosis in serum starved porcine embryonic fibroblasts. Molecular Reproduction and Development 62, 106-112.

Lenardo, M. J. (1991). Interleukin-2 programs mouse $\alpha \beta$ T lymphocytes for apoptosis. Nature, London 353, $858-861$.

Londoño, D. P., Alvarez, J. I, Trujillo, J., Jaramillo, M. M. and Restrepo, B. I. (2002). The inflammatory cell infiltrates in porcine cysticercosis: immunohistochemical analysis during various stages of infection. Veterinary Parasitology 109, 249-259.

Lopes, M. F., Nunes, M. P., Henriques-Pons, A., Giese, N., Morse, H. C. 3rd, Davidson, W. F., Araujo-Jorge, T. C. and DosReis, G. A. (1999). Increased susceptibility of Fas ligand-deficient gld mice to Trypanosoma cruzi infection due to a Th2-biased host immune response. European Fournal of Immunology 29, 81-89.

Martins, G. A., Vieira, L. Q., Cunha, F. Q. and Silva, J. S. (1999). Gamma interferon modulates CD95 (Fas) and CD95 ligand (Fas-L) expression and nitric oxide-induced apoptosis during the acute phase of Trypanosoma cruzi infection: A possible role in immune response control. Infection and Immunity 67, 3864-3871.

Molinari, J. L., Mejia, H., White, A. C. Jr., Garrido, E., Borgonio, V. M., Baig, S. and Tato, P. (2000). Taenia solium: A cysteine protease secreted by metacestodes depletes human CD4 lymphocytes in vitro. Experimental Parasitology 94, 133-142.

Molinari, J. L., Tato, P., Rodriguez, D., Solano, S., Rubio, M. and Sepulveda, J. (1998). Impairment of the inflammatory reaction on implanted Taenia solium metacestodes in mice by a $T$. solium RNA peptide: a scanning electron microscopy study. Parasitology Research 84, 173-180.

Murphy, K. M., Heimberger, A. B. and Loh, Y. (1990). Induction by antigen of intrathymic apoptosis of CD4+ CD8 + TCRlo thymocytes in vivo. Science $\mathbf{2 5 0}$ $1720-1723$.

O’Connell, K. M. and Rogan, M. T. (2000). Apoptosis in human Jurkat $\mathrm{T}$ cells after culture with live Taenia crassiceps cysticerci in vitro. Parasitology 120, 649-655.

Pileri, S. A., Roncador, G., Ceccarelli, C., Piccioli, M., Briskomatis, A., Sabattini, E., Ascani, S., Santini, D., Piccaluga, P. P., Leone, O., Damiani, S., Ercolessi, C., Sandri, F., Pieri, F., Leoncini, L. and Falini, B. (1997). Antigen retrieval techniques in immunohistochemistry: comparison of different methods. Fournal of Pathology 183, 116-123.

Presas, A. M., Robert, L., Jiménez, J. A. and Willms, K. (2005). Apoptosis patterns in experimental Taenia solium and Taenia crassiceps strobilae from golden hamsters. Parasitology Research 96, 1-5.
Resendes, A. R., Majó, N., Segalés, J., Espadamala, J., Mateu, E., Chianini, F., Nofrarías, M. and Domingo, M. (2004). Apoptosis in normal lymphoid organs from healthy normal, conventional pigs at different ages detected by TUNEL and cleaved caspase-3 immunohistochemistry in paraffin-embedded tissues. Veterinary Immunology and Immunopathology 99, 203-213.

Sciutto, E., Fragoso, G., Fleury, A., Laclette, J. P., Sotelo, J., Aluja, A., Vargas, L. and Larralde, C. (2000). Review: Taenia solium disease in humans and pigs: an ancient parasitosis disease rooted in developing countries and emerging as a major health problem of global dimensions. Microbes and Infection 2, 1875-1890.

Silva, E. M., Guillermo, L. V., Ribeiro-Gomes, F. L., De Meis, J., Nunes, M. P., Senra, J. F., Soares, M. B., DosReis, G. A. and Lopes, M. F. (2007). Caspase inhibition reduces lymphocyte apoptosis and improves host immune responses to Trypanosoma cruzi infection. European Fournal of Immunology 37, 738-746.

Silva, R. D., Sotoca, R., Johansson, B., Ludovico, P., Sansonetty, F., Silva, M. T., Peinado, J. M. and Corte-Real, M. (2005). Hyperosmotic stress induces metacaspase- and mitochondria-dependent apoptosis in Saccharomyces cerevisiae. Molecular Microbiology 58, 824-834.

Solano, S., Cortés, I. M., Copitin, N. I., Tato, P. and Molinari, J. L. (2006). Lymphocyte apoptosis in the inflammatory reaction around Taenia solium metacestodes in porcine cysticercosis. Veterinary Parasitology 140, 171-176.

Tato, P., Castro, A. M., Rodriguez, D., Soto, R., Arechavaleta, F. and Molinari, J. L. (1995). Suppression of murine lymphocyte proliferation induced by a small RNA-peptide from the Taenia solium metacestode. Parasitology Research 81, 181-187.

Taylor, C. R., Shi, S. R., Chen, C., Young, L., Yang, C. and Cote, R. J. (1996). Comparative study of antigen retrieval heating methods: microwave, microwave and pressure cooker, autoclave, and steamer. Biotechnic and Histochemistry 71, 263-270.

Uddin, J., Gonzalez, A. E., Gilman, R. H., Garcia, H. H., Verastegui, M., Moore, L. J., Evans, C. A. W., Read, R. C. and Friedland, J. S. (2006).

Neurocysticercal antigens stimulate chemokine secretion from human monocytes via an NF-[kappa]Bdependent pathway. Microbes and Infection $\mathbf{8}$, 1732-1740.

Villa, O. F. and Kuhn, R. E. (1996). Mice infected with the larvae of Taenia crassiceps exhibit a Th2-like immune response with concomitant anergy and downregulation of Th1-associated phenomena. Parasitology 112, 561-570.

White, A. C. Jr., Patrick, H. and Diaz, P. (1995). Asymptomatic neurocysticercosis in a patient with AIDS and cryptococcal meningitis. American Fournal of Medicine 99, 101-102. 